Journal of The Magnetics Society of Japan Vol. 13, Supplement, No. S1 (1989)

(C) 1989 by The Magnetics Society of Japan

\title{
READBACK NOISE AND IMPEDANCE OF THIN FILM HEADS WITH VARIOUS COIL WINDINGS
}

Mako to AIHARA, Mi tsuo SUDA, Kouji YaMAGATA, Hiroshi FUKUI and Yutaka SUGITA

Hi tachi Research Laboratory, Hitachi,Ltd., $4026 \mathrm{Kuji}$, Hitachi, Japan

\begin{abstract}
The noise and the impedance of the thin film head with various coil windings were investigated. The noise can be estimated from the resistance of the head having the small numbers of coil windings by the Nyquist Theorem. With the increase of the coil windings to improve the readback output, the head inductance became not negligible. In the calculation, the frequency characteristics of the head impedance were taken into account. Calculated noise showed good agreement with the measured head noise. Finally, optimum coil windings were discussed.
\end{abstract}

\section{INTRODUCTION}

To optimize coil windings for the maximum reproducing $S / N$, the estimation of the head noise is important in the designing of the thin film head. However, the noise of thin film head with many coil windings has not been studied. The noise of Ni-Zn ferrite heads was studied precisely by Smaller.[1] It is known that the noise of ferrite heads is generated principally by the hysteresis loss, and that of thin film heads is by the coil resistance[2]. However, with increasing the linear density and narrowing the track width, the coil windings of thin film heads have been increasing to improve the readback output. With increasing the coil windings, the inductance of the head increases as same as the resistance, and it should be taken into account for the estimation of the head noise. This research reported here deals with the relation between the head impedance and the noise for various coil windings, and presents the basic understanding of the optimum coil windings for the thin film head. The first part of the paper provides the comparison of the measured head noise with calculated ones by the Nyquist Theorem for the equivalent circuit of the reproducing circuit, which includes the measured head impedance and the input impedance of the reproducing amplifier. After the method of approximation of the impedance is discussed for heads with various coil windings, and the head noise are estimated, the optimum coil windings to maximize $S / N$ of the output of the reproducing system in the magnetic recording system are discussed.

\section{EXPERIMENTAL and RESULTS}

\section{Measurements}

The noise of thin film heads with different number of coil windings, which were connected to a reproducing amplifier, were measured by integrating the noise power spectrum of output of the amplifier measured with a spectrum analyzer (HP3585A) from $500 \mathrm{kHz}$ to $25 \mathrm{MHz}$. Head noises were obtained by subtracting the noise of the reproducing amplifier. Impedance of each head was measured with an impedance analyzer(HP4194A) with relatively low measuring current to eliminate head saturation. Table 1 shows the principal dimensions of the sample heads.

Head Impedance and Noise Figure 1 shows measured noise power

Table 1 Principal dimensions of the samples

\begin{tabular}{|c|c|c|c|c|}
\hline & $\begin{array}{l}\text { Number of } \\
\text { coil turns }\end{array}$ & $\begin{array}{l}\text { Track } \\
\text { idth }(* \mathbb{m})\end{array}$ & $\begin{array}{l}\text { Length of } \\
\text { yoke }(\mu \mathrm{m})\end{array}$ & $\begin{array}{l}\text { Core } \\
\text { idth }(a \mathrm{~m})\end{array}$ \\
\hline A & 17 & 12.0 & 130 & 140 \\
\hline$B$ & 20 & 12.0 & $\uparrow$ & $\uparrow$ \\
\hline C & 21 & 14.5 & - & - \\
\hline $\mathrm{D}$ & 30 & 18.5 & - & - \\
\hline
\end{tabular}




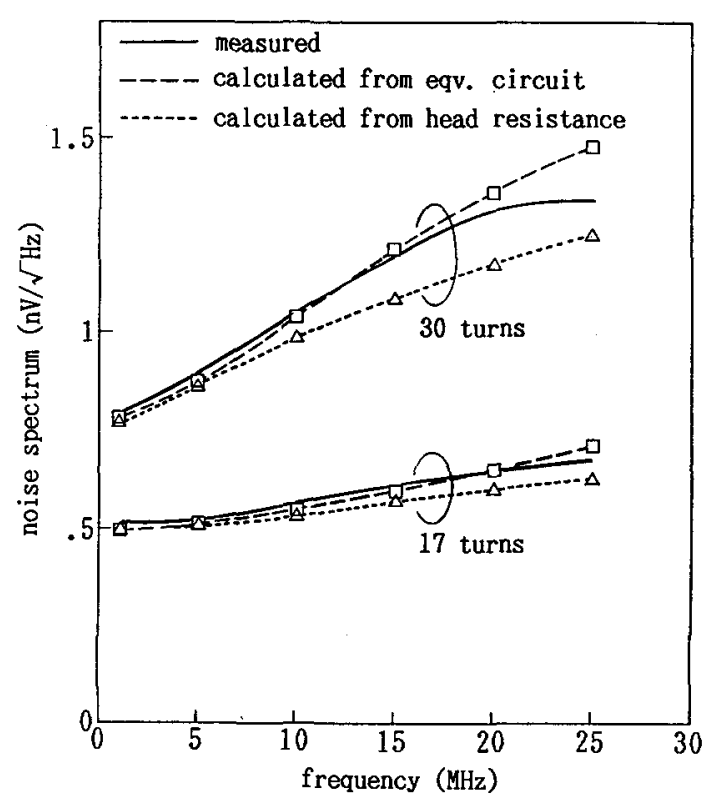

Fig. 1. Noise spectrum of thin film heads.

spectra of heads with 17 and 30 turn coils. The increase of the noise power spectrum with the frequency is small for the head with 17 turn coil, and for the head with 30 turn coil the increase of the spectrum is large. Difference in increase with the frequency should be due to the difference of the frequency characteristics of the head impedance. In this section, the difference of the head noise is related to the head impedance.

The magnitude of the head noise is estimated from the real part of the impedance by the Nyquist theorem, given as follows;

$$
V_{t}^{2}=4 k T \int R(f) d f
$$

where $R$ is the resistance component of the impedance at the frequency $f$, and $k$ is the Boltzmann constant. In the calculation, the real part of the impedance of the equivalent circuit of the reproducing circuit is adopted. which includes the head impedance and the input impedance of the reproducing amplifier as shown in figure 2 .

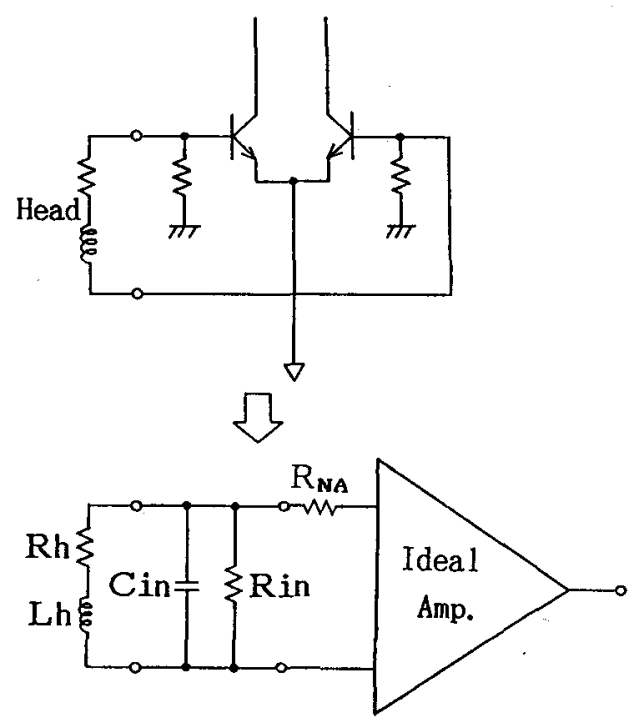

Fig. 2. Equivalent circuit of reproducing circuit includes input capacitance and resistance of a reproducing amplifier, and the head impedance. Rna is a equivalent noise resistance of the reproducing amplifier.

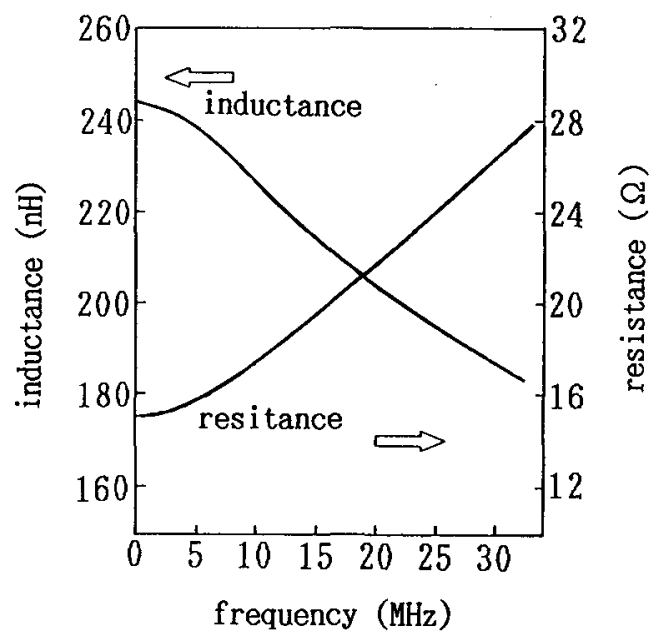

Fig. 3. Head impedance change with frequency. 
Figure 3 shows the change of the head impedance with the frequency. The real part of the head impedance above several megahertz increases proportionally to the frequency. The imaginary part of the head impedance decreases above $3 \mathrm{MHz}$. These change of the impedance are due to the magnetic loss of the head core.

The noise power spectra calculated from the equivalent circuit are shown by the broken line in Figure 1. The noise power spectra calculated only from the real part of the head impedance are also shown in Figure 1, and agree with the measured noise power spectrum. This means the increase of the head noise is mainly caused by the magnetic loss of the core. The spectra calculated from the equivalent circuit which includes the input impedance of the first-stage amplifier show better agreement with the measured spectra, than those from only the real part of the head imisdance.

Figure 4 shows the total noises integrated the spectra obtained from the

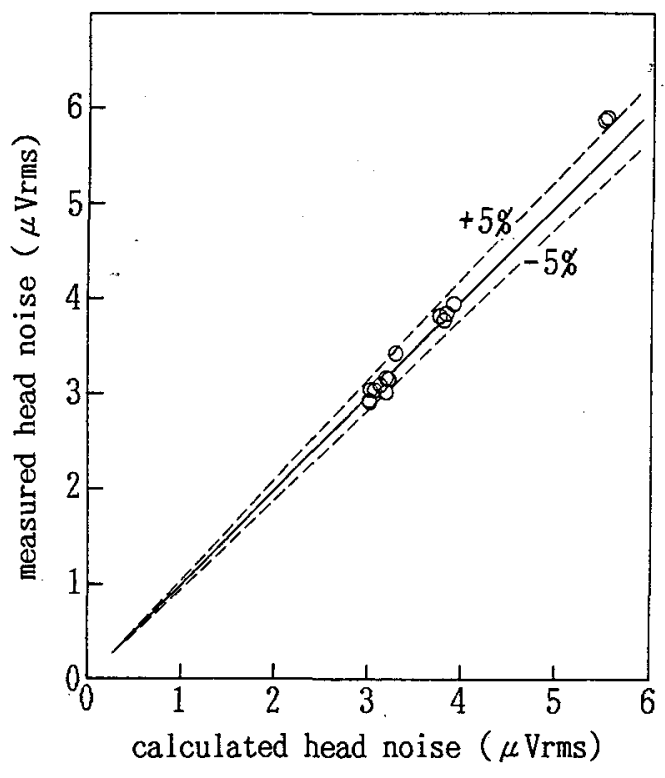

Fig. 4. Comparison between measured, and calculated head noise from measured head impedances. equivalent circuit for heads with coil windings of $17,20,21$, and 30 , and they agree with the measured ones.

Estimation of Head Impedance and Noise If the head impedance and its frequency characteristics are predicted, the head noise can be calculated as described in the previous section. In this section, we estimate the head impedance and the noise.

The increase of the real part and the decrease of the imaginary part with the frequency are caused by the eddy current loss and the hysteresis loss of the magnetic layer of the head. However the effects of these losses for the thin film head are not clear.

For simplicity, the head impedance is approximated with linear functions. Figure 5 shows the increase rate of the real part with the frequency. The gradient is proportional to the magnitude of the inductance of each head. That is, the head with larger inductance have larger

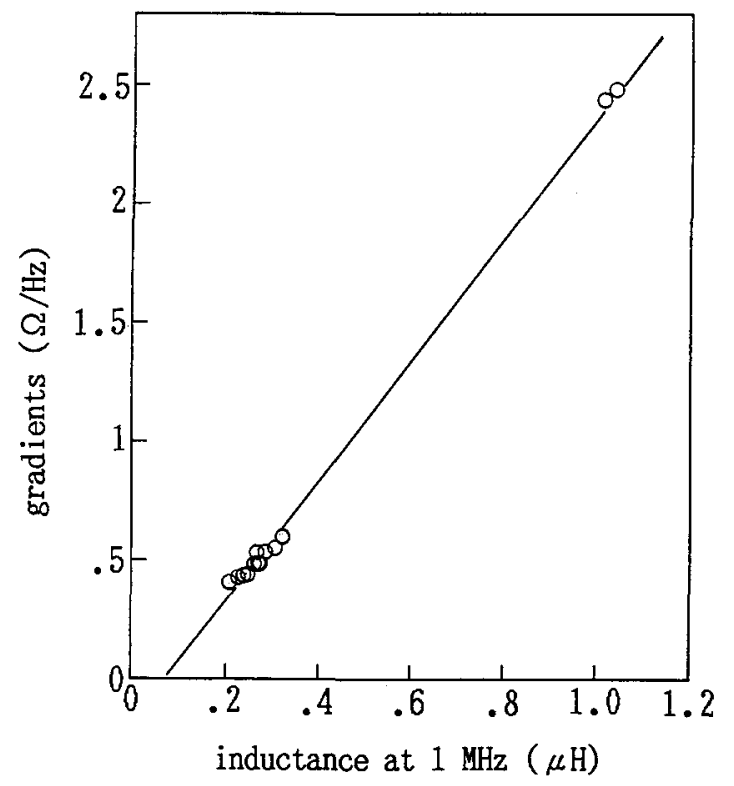

Fig. 5. Gradients of resistance increase with frequency as function of head inductance. 
increase at its real part with the frequency. The rate of the decrease of the head inductance also has good correlation to the magnitude of the head inductance at the low frequency. That is, the head with larger inductance shows larger decrease of the inductance with the frequency. We introduce lerms of the inductance of the head core into following functions of the frequency characteristics of the head impedance. We assume the increase rate of the real part and the decrease rate of the imaginary part to be proportional to the head core inductance at the low frequency.

$$
\begin{array}{ll}
R(f)=R_{100} & f<3 M H z \quad(2) \\
R(f)=R_{100}+K_{1}\left(L_{100}-L_{\text {coil } 10}\right) f & f \geqq 3 M H z \quad(3) \\
L(f)=L_{100} & f<3 M H z(4) \\
L(f)=L_{100}-K_{2}\left(L_{100}-L_{\text {coil } 10}\right) f & f \geqq 3 M H z \quad(5)
\end{array}
$$

Here, the inductance of the head core is obtained by subtracting I.coilo, which is the inductance of the coil without the head core, from the head inductance Llow at the low frequency.

To verify these approximation, the head noise are calculated with the approximate functions for the heads measured in previous section. Here, the input impedance of the reproducing amplifier is assumed to be constant, and to have no frequency dependence. The method of the calculation is the same as the previous section, the integration of the noise spectrum of the equivalent circuitry, which includes the head impedance and the input impedance of the reproducing amplifier.

Figure 6 shows approximated and measured noise of the heads. The calculated head noise with the approximated head impedance shows good agreement with the measured ones.

Coil Windings and Signal to Noise Ratio The noise $N$ at the output of the reproducing amplifier is

$$
\mathrm{N}=\sqrt{\mathrm{N}_{\mathrm{d}}^{2}+\mathrm{N}_{\mathrm{h}}^{2}+\mathrm{N}_{\mathrm{a}}^{2}}
$$

Where, Nd is the disk noise, Nh is the head noise, and $\mathrm{Na}$ is the amplifier noise. Then, $S / N$ of head with a fixed magnetic path length is

$$
\mathrm{S} / \mathrm{N}=\frac{\mathrm{E}}{\beta \sqrt{\mathrm{N}_{\mathrm{d}}^{2}+\mathrm{N}_{\mathrm{h}}^{2}+\mathrm{N}_{\mathrm{a}}^{2}}}
$$

where, $E$ is the output of the amplifier and $b$ is constant.

Figure 7 shows the assumed change of the reproducing $\mathrm{signal}$ and noises with the increase of the coil windings. Here, $N d$ is assumed to be proportional to the number of coil windings, as same as the reproducing signal. The amplifier noise is constant, when the data rate and the bandwidth are constant.

Equation (7) is rewritten as follows;

$$
\mathrm{S} / \mathrm{N}=\frac{\mathrm{E}_{0}}{\beta \sqrt{\mathrm{N}_{\mathrm{d} 0}^{2}+\frac{\mathrm{N}_{\mathrm{a}}^{2}+\mathrm{N}_{\mathrm{h}}^{2}}{\mathrm{n}^{2}}}}
$$

Where, $n$; number of the coil windings

NdO ;Disk noise per one turn of the coil 1

EO ; output per one turn of the coil Then, $S / N$ takes the maximum for the coil windings that makes the second term in the square root in the denominator is the minimum. The disk noise does not affect the coil windings for maximum of $S / N$.

Based on a few assumptions, the coil resistance is proportional to the square of coil windings for the fixed magnetic path length. Therefore, if the head noise is assumed to be generated only by the coil resistance, the second term in the square root decreases with coil windings, and $S / N$ saturate. With the effect of loss of the magnetic layer, however, the head noise is larger than this assumption, $\mathrm{S} / \mathrm{N}$ decrease and takes a peak at the optimum coil windings.

\section{CONCLUSIONS}

At the thin film heads with many coil windings, the head inductance has been 
becoming not negligible to estimate the head noise. The head noise was obtained from the real part of the impedance of the reproducing circuit by the Nyquist theorem. In the calculation, the input impedance of the first stage amplifier of the reproducing circuitry should be taken into account. Calculated noise showed good agreement with the measured head noise. The frequency characteristics of the real and imaginary parts of the head impedance depended the inductance of the head in the low frequency. By approximating the frequency dependence of each parts of the head impedance, the head noise could be estimated. With increasing the coil windings, due to the effect of the inductance of the head, the head noise. increased, and there was optimum coil winding numbers to maximize $S / N$ of the reproducing system.

\section{ACKNOWI.EDGMENTS}

We would like to appreciate Messrs. Aoi and Koizumi of Central Research Laboratory of Hitachi for their useful discussion on the head noise. Authors would like to extend our thanks to Messrs. Kawakami, Kameyama and other people in Odawara works of Hitachi, for their assistance throughout this work.

\section{References}

[1]P.Smaller, I EEE Trans. Magn., MAG-1, 357 (1969)

[2]C.I) Hee and E.D.Daniel, "Magnetic Recording Vol.1",340 344(1987)

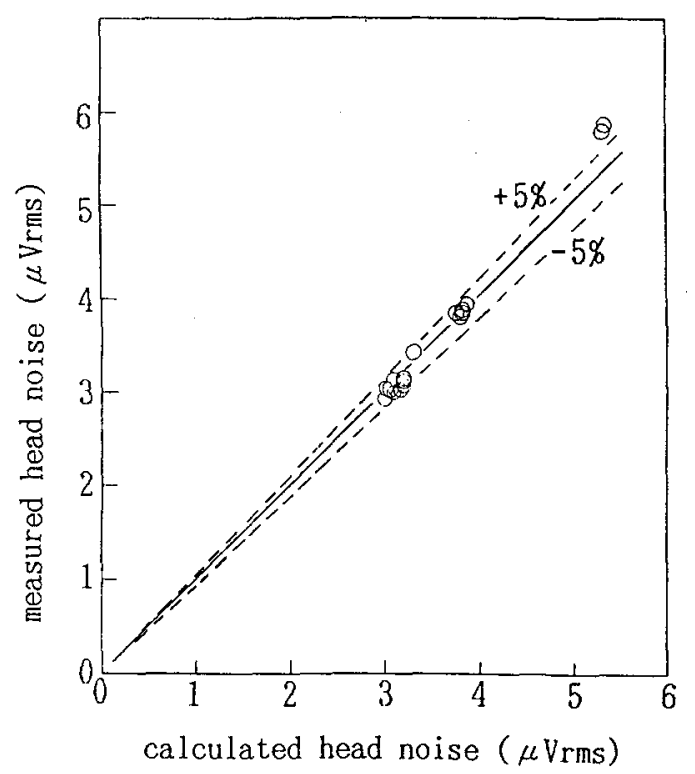

Fig. 6. Comparison between measured, and calculated head noise from approximated head impedances.

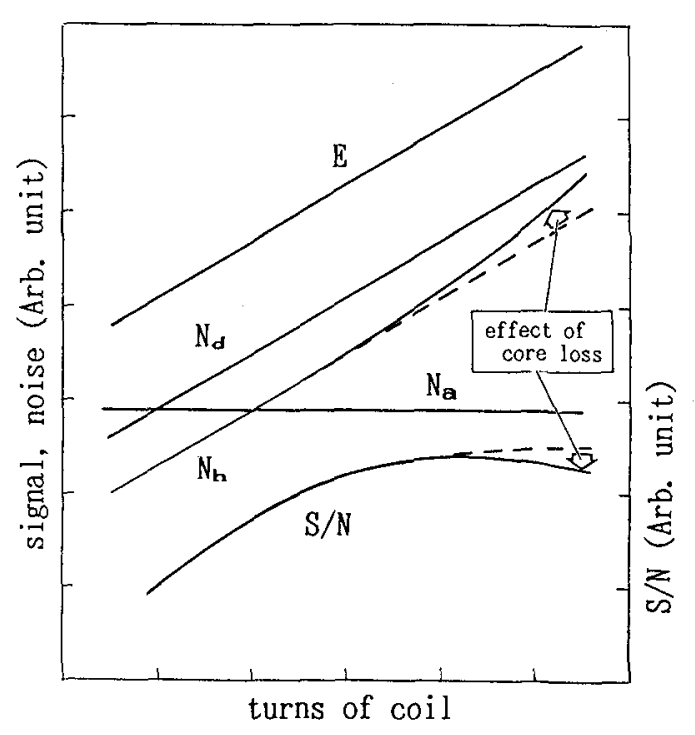

Fig. 7. Change of noises with coil turns. 